\title{
Author Correction: A proteomic landscape of diffuse-type gastric cancer
}

Sai Ge (10 1,2, Xia Xia ${ }^{1}$, Chen Ding ${ }^{1,3}$, Bei Zhen ${ }^{1}$, Quan Zhou ${ }^{1}$, Jinwen Feng ${ }^{1,4}$, Jiajia Yuan ${ }^{1,2}$, Rui Chen ${ }^{5}$, Yumei Li ${ }^{5}$, Zhongqi Ge ${ }^{5}$, Jiafu $\mathrm{Ji}^{2}$, Lianhai Zhang (1) ${ }^{2}$, Jiayuan Wang ${ }^{2}$, Zhongwu $\mathrm{Li}^{2}$, Yumei Lai ${ }^{2}$, Ying $\mathrm{Hu}^{2}$, Yanyan $\mathrm{Li}^{2}$, Yilin $\mathrm{Li}^{2}$, Jing $\mathrm{Gao}^{2}$, Lin Chen ${ }^{6}$, Jianming $\mathrm{Xu}{ }^{7}$, Chunchao Zhang ${ }^{8}$, Sung Yun Jung ${ }^{8}$, Jong Min Choi ${ }^{8}$, Antrix Jain ${ }^{8}$,

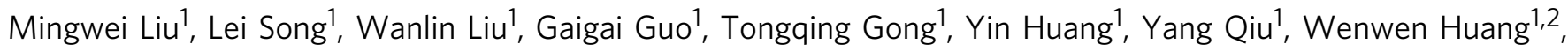
Tieliu Shi ${ }^{4}$, Weimin Zhu1, Yi Wang ${ }^{1,8}$, Fuchu He ${ }^{1,3}$, Lin Shen ${ }^{1,2}$ \& Jun Qin ${ }^{1,3,8}$

Correction to: Nature Communications https://doi.org/10.1038/s41467-018-03121-2, published online: 08 March 2018.

The original version of this article contained an error in the email address of the corresponding author Jun Qin. The correct email is jqin1965@126.com. The error has been corrected in the HTML and PDF versions of the article.

Published online: 08 May 2018

\begin{abstract}
ceproduction in any medium or format, as long as you give appropriate credit to the original author(s) and the source, provide a link to the Creative Commons license, and indicate if changes were made. The images or other third party material in this article are included in the article's Creative Commons license, unless indicated otherwise in a credit line to the material. If material is not included in the article's Creative Commons license and your intended use is not permitted by statutory regulation or exceeds the permitted use, you will need to obtain permission directly from the copyright holder. To view a copy of this license, visit http://creativecommons.org/licenses/by/4.0/.
\end{abstract}

(C) The Author(s) 2018

\footnotetext{
${ }^{1}$ The Joint Laboratory of Translational Medicine, National Center for Protein Sciences (Beijing) and Peking University Cancer Hospital, State Key Laboratory of Proteomics, Institute of Lifeomics, 102206 Beijing, China. ${ }^{2}$ Key Laboratory of Carcinogenesis and Translational Research (Ministry of Education), Peking University Cancer Hospital \& Institute, 100142 Beijing, China. ${ }^{3}$ State Key Laboratory of Genetic Engineering, Human Phenome Institute, Institutes of Biomedical Sciences, and School of Life Sciences, Zhongshan Hospital, Fudan University, 200433 Shanghai, China. ${ }^{4}$ Center for Bioinformatics, East China Normal University, 200241 Shanghai, China. ${ }^{5}$ Human Genome Sequencing Center, Department of Molecular and Human Genetics, Baylor College of Medicine, Houston, TX 77030, USA. ${ }^{6}$ General Hospital of Chinese People's Liberation Army, 100853 Beijing, China. ${ }^{7}$ Affiliated Hospital of Academy of Military Medical Sciences, 100071 Beijing, China. ${ }^{8}$ Alkek Center for Molecular Discovery, Verna and Marrs McLean Department of Biochemistry and Molecular Biology, Department of Molecular and Cellular Biology, Baylor College of Medicine, Houston, TX 77030, USA. These authors contributed equally: Sai Ge, Xia Xia, Chen Ding, Bei Zhen. Correspondence and requests for materials should be addressed to F.H. (email: hefc@nic.bmi.ac.cn) or to L.S. (email: linshenpku@163.com) or to J.Q. (email: jain1965@126.com)
} 